\title{
Egyptian Association of Vascular Biology and Atherosclerosis (EAVA) consensus on the usage of proprotein convertase subtilisin/kexin type 9 (PCSK9) inhibitors
}

\author{
Ashraf Reda ${ }^{1 *}$, Ahmed Shawky Elserafy ${ }^{2}$, Elsayed Farag ${ }^{3}$, Tamer Mostafa $^{3}$, Nabil Farag ${ }^{2}$, Atef Elbahry ${ }^{4}$, \\ Osama Sanad ${ }^{5}$, Ahmed Bendary ${ }^{5^{*}}$ D, Ahmed Elkersh ${ }^{1}$, Mohammed Selim ${ }^{6}$, Morad Beshay ${ }^{1}$ and Hazem Khamis ${ }^{7}$
}

\begin{abstract}
Background: The current expert view of the PCSK9 inhibitors' use in Egypt is still ambiguous.

Main body: Hyperlipidemia is an important, if not the most important, risk factor for the occurrence of atherosclerosis worldwide. Egypt is the most populous country in the Middle East and North Africa and has > 15\% of the cardiovascular deaths in the region. The burden of dyslipidemia as seen in the recently published CardioRisk project conducted throughout Egypt shows a high prevalence of dyslipidemia as a risk factor that is still reaching up to $71 \%$ in female participants. Reaching the targets for LDL lowering, and thus control of hyperlipidemia, is quite often very difficult especially with the update of the last ESC guidelines. With the advent of PCSK9 inhibitors, the control rate of patients, reduction of cardiac major adverse events, and mortality have been improved. However, Egypt is not considered a rich country on the grounds of annual income, and this raises a concern on which patients would benefit from these expensive medications. Revising the randomized control trials, we analyzed the data that would enable us to control LDL in those patients, at risk, to obtain simple clear indications for the use of these rather expensive medications.

Conclusion: We recommend the use of PCSK9 inhibitors in addition to statins \pm ezetimibe in patients with ASCVD, by definition at very high risk; patients with ASCVD at very high risk who do not tolerate appropriate doses of at least three statins; and familial hypercholesterolaemia patients with clinically diagnosed ASCVD, at very high cardiovascular risk.
\end{abstract}

Keywords: Egypt, Dyslipidemia, PCSK-9

\section{Background}

Egypt is the most populous country in the Middle East and North Africa and has $>15 \%$ of the cardiovascular deaths in the region. The burden of dyslipidemia as seen in the recently published CardioRisk project conducted

\footnotetext{
*Correspondence: ashrafreda5555@gmail.com; dr_a_bendary@hotmail.com; ahmed.bendari@fmed.bu.edu.eg

${ }^{1}$ Cardiology Department, Faculty of Medicine, Menofia University, Menofia, Egypt

${ }^{5}$ Cardiology Department, Faculty of Medicine, Benha University, Benha, Egypt Full list of author information is available at the end of the article
}

throughout Egypt shows a high prevalence of dyslipidemia as a risk factor that is still reaching up to $71 \%$ in female participants. Reaching the targets for LDL lowering, and thus control of hyperlipidemia, is quite often very difficult especially with the update of the last ESC guidelines. With the advent of PCSK9 inhibitors, the control rate of patients, reduction of cardiac major adverse events, and mortality have been improved. However, Egypt is not considered a rich country on the grounds of annual income, and this raises a concern on which patients would benefit from these expensive 
medications. Accordingly, the Egyptian Association of Vascular Biology and Atherosclerosis (EAVA) took the responsibility of providing an expert consensus on the future and potential use of these medications in Egyptian patients with dyslipidemia.

\section{Main text}

The role of low-density lipoprotein cholesterol lowering in atherosclerotic cardiovascular disease prevention

Among all lipid profile parameters, low-density lipoprotein cholesterol (LDL-C) remains the primary analyte and treatment target according to recent guidelines [1]. The causal role of LDL-C in the induction and progression of the atherosclerotic process is unquestionable [2]. Since the introduction of the 3-hydroxymethyl glutaryl co-enzyme A (HMG-co-A) inhibitors [statins] into clinical practice, there has been a robust accumulating evidence that LDL-C lowering results in a reduction of incident ASCVD events (for every $1 \mathrm{mmol} / \mathrm{l}[39 \mathrm{mg} / \mathrm{dl}]$ lowering, there is a corresponding $22 \%$ relative risk reduction (RRR) of incident ASCD events) [3]. Moreover, the current emphasis is on the concept that "even the lower LDL-C, even the better"; this is because new evidence coming from the trials of non-statin lipidlowering therapy points to an additional benefit with further LDL-C lowering beyond target levels previously considered adequate. Ezetimibe (a cholesterol absorption inhibitor) added to simvastatin resulted in a significant $6.4 \%$ RRR in the outcome in the IMPROVE-IT trial [4]. Of note, the mean LDL-C achieved in the arm of ezetimibe-simvastatin was $54 \mathrm{mg} / \mathrm{dl}$ [4]. The addition of ezetimibe to statins has another potential to reduce the incidence of statin intolerance such as the occurrence of myalgias [5]. Then, the trials of proprotein convertase subtilisin/kexin type 9 (PCSK-9) inhibitors came, which corroborated the notion that lower is better. Evolocumab in the FOURIER trial [6] added to maximally tolerated statin therapy in patients with stable established ASCVD (achieving a mean LDL-C level of merely 30 $\mathrm{mg} / \mathrm{dl}$ ) safely resulted in a significant $15 \%$ RRR in a composite ASCVD events endpoint. Alirocumab resulted in a similar magnitude of benefit (with no safety issues) in the ODYSSEY-OUTCOMES trial [7] which examined relatively more unstable patients (those who sustained an acute coronary syndrome (ACS) in the past 12 months). Of note, the ODYSSEY results (in subgroups' analyses) were consistent among patients with DM and ACS [8] in addition to patients with stroke [9]. Taken together, these data have prompted the 2019 ESC dyslipidemia guideline committee to lower the LDL-C treatment goals across all risk categories, with even a class IIb consideration given to a level of $40 \mathrm{mg} / \mathrm{dl}$ in very high-risk patients who are still experiencing ASCVD events [1].

\section{Reaching the LDL-C goals is difficult}

According to the EUROASPIRE-V program [10], which included 8261 patients surveyed after hospitalization for ACS or coronary revascularization in 131 centers covering 27 European countries, about $60 \%$ were on highintensity statins. Only $36 \%$ of them achieved an LDL-C level of $<70 \mathrm{mg} / \mathrm{dl}$. The Egyptian data showed that the situation might be worse; among very high-risk patients receiving maximally tolerated statin therapy, LDL-C treatment goals are reached in $22.3 \%$ of them [11]. An explanation for these poor figures remains elusive; however, it seems that statins' effect on LDL-C lowering has a "ceiling effect," meaning that the highest dose of any statin cannot attain more than 55-60\% reduction of baseline LDL-C levels [12]. The problem becomes more complex if we consider that LDL-C treatment goals are becoming furtherly lower in the newer guidelines [1]. This clearly sets the stage for non-statin lipid-lowering therapies to be key players in this field.

\section{Premature coronary artery disease and familial hypercholesterolemia: current status in Egypt}

In recent years, there has been increasing recognition of the problem of familial hypercholesterolemia (FH). The European Atherosclerosis Society (EAS) in collaboration with the Egyptian Association of Vascular Biology and Atherosclerosis (EAVA) and other national societies published an international report about the current status of FH in over 60 countries [13]. Data from the Egyptian CardioRisk project [14] point to a $51 \%$ estimated prevalence of premature coronary artery disease (CAD) (defined as ACS before age 55 years in males and 65 years in females) due to noticeably larger burden of traditional risk factors especially smoking and possibly familial hypercholesterolemia. The Egyptian familial hypercholesterolemia research forum is a national initiative powered by EAVA in collaboration with EAS. Its preliminary results [15] showed that among cases with suspected FH $(31 \%, 12 \%$, and $57 \%$ in the definite, probable, and possible categories respectively according to the Dutch Lipid Clinic Network criteria), mean total cholesterol was $339 \pm 100 \mathrm{mg} / \mathrm{dl}$ and mean LDL-C was $249 \pm 98 \mathrm{mg} / \mathrm{dl}$. It is imperative to note that these levels are on the background of conventional lipid-lowering therapy (41\% statin monotherapy and 59\% combination with ezetimibe). Unfortunately, only one patient received lipoprotein apheresis and 6 patients fulfilled for a PCSK9 inhibitor. The above figures reflect the significant unmet need for a better and a more effective strategy to treat these high-risk patients (including the use of PCSK-9 inhibitors which, after a major price cut [16], are thought now to meet the accepted cost-effectiveness international thresholds [17]). 


\section{Proprotein convertase subtilisin/kexin type 9 becomes recognized}

Many reports have revealed that PCSK-9 has a critical role in the life cycle of the LDL receptors [18]. LDL receptors are clustered in clathrin-coated pits on the surface of hepatocytes. After binding to LDL, an endocytic vesicle is produced. In the endosome, the LDL receptor can undergo a conformational change with release of the LDL particle; the LDL receptor is then recycled back to the surface of the hepatocyte to extract more LDL from the circulation. Of note, LDL receptors can be recirculated approximately 100 times. On the other hand, the LDL particle is transferred to the lysosome where it is degraded. PCSK9 is produced by hepatocytes and attaches to the epidermal growth factor-like repeat $\mathrm{A}$ (EGF-A) domain of the LDL receptor. When the PCSK9-LDL receptor complex is internalized, the presence of PCSK9 prevents the conformational change in the LDL receptor, and the LDL receptor then passes together with LDL to the lysosome, where it is destroyed [18].

\section{PCSK9 monoclonal inhibitors}

The advent of monoclonal antibodies against PCSK9 leads the way to a novel mechanism of management of hypercholesterolemia [19]. Evolocumab can be prescribed at a dose of $140 \mathrm{mg}$ every 2 weeks [20]. In phase III clinical trials, evolocumab decreased plasma LDL-C levels by approximately $60 \%$ whether given as a monotherapy, added to statin therapy, given to patients who were statin-intolerant, or given to patients with heterozygous FH [21-25]. Overall, evolocumab reduced LDLC levels regardless of baseline plasma PCSK9 levels [26]. In patients with homozygous $\mathrm{FH}$, the picture was variable. Thus, additive management plans are needed for patients with homozygous $\mathrm{FH}$, especially those with LDLR-negative alleles.

Alirocumab has been given with different regimens in phase III clinical trials. One regimen was an escalating dose that was based on attained reductions in plasma LDL-C levels: patients would start at $75 \mathrm{mg}$ of alirocumab every 2 weeks and then the dose would be escalated to $150 \mathrm{mg}$ every 2 weeks if the LDL-C level was $\geq 70 \mathrm{mg} /$ dl. In general, this approach resulted in LDL-C level reductions of $45-50 \%$ whether given as monotherapy, added to statin therapy, or given to patients who were statin-intolerant [27-30]. The maximum dose of alirocumab in these trials, $150 \mathrm{mg}$ every 2 weeks, decreased plasma LDL-C levels by about 60\% [31], which is like evolocumab. Data concluded that in patients with heterozygous $\mathrm{FH}$, treatment with alirocumab decreased LDL-C levels by about 40-60\% [32, 33]. In patients with homozygous $\mathrm{FH}$, the changes in LDL-C levels demonstrated with alirocumab therapy ranged from a $7 \%$ rise to a $64 \%$ decline according to the genotype of the patient [34].

\section{Trials that changed the guidelines}

The earliest study to report cardiovascular outcomes with the use of PCSK-9 inhibitors was the FOURIER trial [35]. More than 27,000 patients with established atherosclerotic cardiovascular disease (ASCVD) were enrolled in the trial. Patients were eligible for inclusion if they had an LDL-C level $\geq 70 \mathrm{mg} / \mathrm{dl}$ or a non-HDLcholesterol level $\geq 100 \mathrm{mg} / \mathrm{dl}$ and taking optimal lipidlowering therapy, specifically high-intensity statins, with or without ezetimibe. Patients were allocated to receive evolocumab (either $140 \mathrm{mg}$ every 2 weeks or $420 \mathrm{mg}$ every month, according to the patient's preference) or matching placebo injections. Evolocumab decreased LDL-C levels by $59 \%$ relative to placebo, with a mean absolute decrease of $56 \mathrm{mg} / \mathrm{dl}$ to a median level of 30 $\mathrm{mg} / \mathrm{dl}$, and this reduction was stable over time. Significant reductions $(21-27 \%)$ were reported in the risks of fatal or nonfatal MI, fatal or nonfatal stroke, and coronary revascularization versus placebo [6]. No impact was observed on cardiovascular death or hospital admissions for unstable angina [6]. As seen in various clinical trials of statins, the translation of LDL-C lowering into clinical benefit is a matter of time. Specifically, the clinical risk reduction per millimoles per liter of LDL-C lowering is much less in the first year of statin therapy compared with the following years [36]. Adverse events tended to be similar between evolocumab and placebo. No higher risk of diabetes was observed with evolocumab relative to placebo even when patients were prediabetics at baseline, which is the subgroup of patients in whom the heightened risk of diabetes with statin therapy seems to be largely confined [37, 38].

When exploring neurocognitive events, the EBBINGHAUS trial was a specific, neurocognitive sub-study conducted on 1974 subjects from the FOURIER trial [39]. No statistically significant differences in cognitive function were noted. Moreover, an exploratory analysis found no consistent correlation between LDL-C levels and cognitive changes [40]. Also, thanks to the fact that evolocumab is a fully human antibody, no patients developed neutralizing antibodies [6].

The ODYSSEY Outcomes trial enrolled a total of 18, 924 patients who were recruited 1 to 12 months after hospitalization for MI or unstable angina. Inclusion criteria were LDL-C level $\geq 70 \mathrm{mg} / \mathrm{dl}$, non-HDL-C level $\geq$ $100 \mathrm{mg} / \mathrm{dl}$, or APOB level $\geq 80 \mathrm{mg} / \mathrm{dl}$. Patients were required to be on high-intensity statin therapy (namely, atorvastatin $\geq 40 \mathrm{mg}$ or rosuvastatin $\geq 20 \mathrm{mg}$ daily) or receiving the maximum tolerable dose of one of those statins. Patients were randomly allocated to receive alirocumab (75 mg or $150 \mathrm{mg}$ every 2 weeks) or matching 
subcutaneous placebo injections. The dose of alirocumab was escalated to achieve an LDL-C level of $25-50 \mathrm{mg} / \mathrm{dl}$, and alirocumab was halted when the LDL-C level was persistently $<15 \mathrm{mg} / \mathrm{dl}$. The primary efficacy outcome was the composite of CHD death, MI, ischemic stroke, or hospitalization for unstable angina (requiring ECG changes and angiographic evidence of clinically significant disease) [7]. Alirocumab decreased LDL-C levels by $57 \%$ at 4 weeks relative to placebo; however, this effect was attenuated with time, with reductions of $50 \%$ at 1 year and $36 \%$ at the end of the study, due to the downtitration algorithm [7]. The median follow-up was 2.8 years. Over the course of the study, alirocumab showed a significant reduction in the risk of the primary outcome by $15 \%$ compared to placebo (HR $0.85,95 \%$ CI $0.78-0.93, P=0.003)$. The impact of alirocumab on the individual components of the composite endpoint, including coronary heart death, was directionally homogenous, i.e., there was no heterogenous treatment effect. A "nominal" 15\% reduction (HR 0.85, 95\% CI 0.73-0.98) in all-cause mortality with alirocumab treatment compared with placebo was also noticed. A further analysis according to baseline LDL-C level showed a non-monotonic pattern of risk reduction of the primary efficacy endpoint with alirocumab, with a HR 0.86 (95\% CI 0.74-1.01) in the groups of patients with a baseline LDL-C level < $80 \mathrm{mg} / \mathrm{dl}$, HR 0.96 (95\% CI 0.82-1.14) in patients with a baseline LDL-C level $80-100 \mathrm{mg} / \mathrm{dl}$, and HR 0.76 (95\% CI 0.65-0.87) in patients with a baseline LDL-C level $\geq 100 \mathrm{mg} / \mathrm{dl}$ [7]. However, given the idea that the protocol-mandated reduction of therapy on the basis of achieved LDL-C levels, patients assigned to alirocumab treatment who had a lower LDL-C level at baseline were more likely to have their alirocumab dose reduced or stopped, thereby offsetting the useful impact of alirocumab in these patients. The safety data seemed

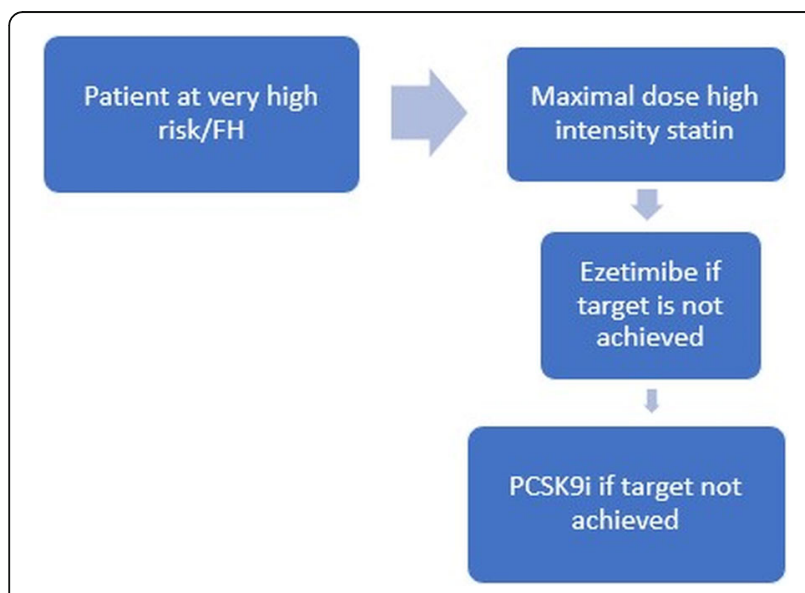

Fig. 1 Proposed algorithm for the starting of PCSK9 inhibitors in very high-risk patients and in familial hypercholesterolemia

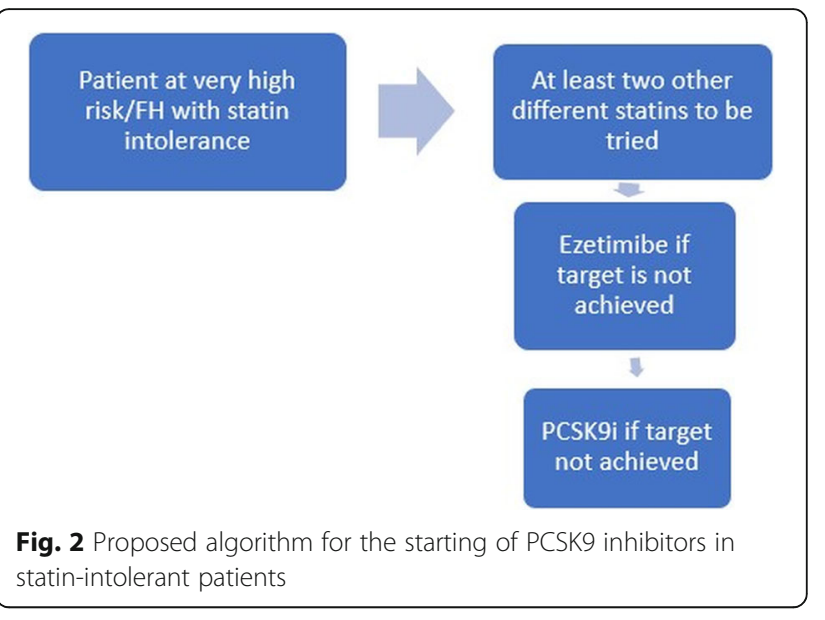

equally reassuring as that seen in the FOURIER trial, with no significant differences in elevations of creatine kinase or aminotransferase levels in the plasma and no excess in the occurrence of new-onset diabetes, cataracts, or neurocognitive declines [7].

\section{Conclusions}

Having revised the evidence from the cardiovascular outcome studies with PCSK9 inhibitors as well as from the available Egyptian data as we showed, we can conclude that addition of a PCSK9 inhibitor should be considered in (1) patients with ASCVD, by definition at very high risk (Fig. 1); (2) patients with ASCVD and at very high risk who do not tolerate appropriate doses of at least three statins (Fig. 2); and (3) familial hypercholesterolemia patients with clinically diagnosed ASCVD at high cardiovascular risk or for primary prevention if with very high cardiovascular risk (provided that the patient is on the maximally tolerated statin dose in addition to combination with Ezetimibe, adopting the same targets as in the 2019 ESC guidelines).

\section{Abbreviations}

ACS: Acute coronary syndrome; ASCVD: Atherosclerotic cardiovascular disease; CAD: Coronary artery disease; EAS: European Atherosclerosis Society; EAVA: Egyptian Association for Vascular Biology and Atherosclerosis; FH: Familial hypercholesterolemia; LDL-C: Low-density lipoprotein cholesterol; PCSK-9: Proprotein convertase subtilisin/kexin type 9

\section{Acknowledgements}

We acknowledge the support of the Egyptian Association of Vascular Biology and Atherosclerosis (EAVA).

\section{Authors' contributions}

AR conceived and designed the consensus and critically revised the manuscript. $A S$ and $A B$ were the major contributors to the initial draft and writing. EF, OS, NF, TM, AE, HK, MS, MB, and AE helped in drafting the manuscript. All authors have read and approved the final manuscript. 


\section{Availability of data and materials}

All data are available upon request from the corresponding author(s).

\section{Ethics approval and consent to participate}

Not applicable.

\section{Consent for publication}

Not applicable.

\section{Competing interests}

The authors declare no conflict of interest.

\section{Author details}

${ }^{1}$ Cardiology Department, Faculty of Medicine, Menofia University, Menofia, Egypt. ${ }^{2}$ Cardiology Department, Faculty of Medicine, Ain Shams University, Cairo, Egypt. ${ }^{3}$ Cardiology Department, Faculty of Medicine, Zagazig University, Zagazig, Egypt. ${ }^{4}$ Cardiology Unit, Port Fouad Centre, Port Fouad, Egypt. ${ }^{5}$ Cardiology Department, Faculty of Medicine, Benha University, Benha, Egypt. ${ }^{6}$ National Heart Institute, Giza, Egypt. ${ }^{7}$ Cardiology Department, Faculty of Medicine, 6th of October University, Cairo, Egypt.

\section{Received: 19 February 2020 Accepted: 27 April 2020} Published online: 18 May 2020

\section{References}

1. Mach F, Baigent C, Catapano AL et al (2019) 2019 ESC/EAS guidelines for the management of dyslipidaemias: lipid modification to reduce cardiovascular risk. Atherosclerosis 290:140-205

2. Ference BA, Ginsberg HN, Graham I et al (2017) Low-density lipoproteins cause atherosclerotic cardiovascular disease. 1. Evidence from genetic, epidemiologic, and clinical studies. A consensus statement from the European atherosclerosis society consensus panel. Eur Heart J 38(32):24592472

3. Baigent C, Blackwell L, Emberson J et al (2010) Efficacy and safety of more intensive lowering of LDL cholesterol: a meta-analysis of data from 170,000 participants in 26 randomized trials. Lancet 376:1670-1681

4. Cannon CP, Blazing MA, Giugliano RP et al (2015) Ezetimibe added to statin therapy after acute coronary syndromes. N Engl J Med 372(25):2387-2397

5. Ruscica M, Reiner Z, Sirtori CR (2019) Can we further optimize statin therapy to increase tolerability? Expert Opin Drug Discov 14(9):843-847

6. Sabatine MS, Giugliano RP, Keech AC et al (2017) Evolocumab and clinical outcomes in patients with cardiovascular disease. N Engl J Med 376(18): 1713-1722

7. Schwartz GG, Steg PG, Szarek M et al (2018) Alirocumab and cardiovascular outcomes after acute coronary syndrome. N Engl J Med 379(22):2097-2107

8. Ray KK, Colhoun HM, Szarek M et al (2019) Effects of alirocumab on cardiovascular and metabolic outcomes after acute coronary syndrome in patients with or without diabetes: a prespecified analysis of the ODYSSEY OUTCOMES randomised controlled trial. Lancet Diab Endocrinol 7(8):618628

9. Jukema JW, Zijlstra LE, Bhatt DL et al (2019) Effect of alirocumab on stroke in ODYSSEY OUTCOMES. Circulation 140(25):2054-2062

10. Kotseva K, De Backer G, De Bacquer D et al (2019) Lifestyle and impact on cardiovascular risk factor control in coronary patients across 27 countries: results from the European Society of Cardiology ESC-EORP EUROASPIRE V registry. Eur J Prev Cardiol 26(8):824-835

11. Reda A, Abdel-Rehim AA, Etman A, Afifi OSA (2014) Centralized pan-Middle East survey on the under-treatment of hypercholesterolemia: results from the CEPHEUS study in Egypt. Cardiol Therapy 3(1-2):27-40

12. Weng TC, Yang YHK, Lin SJ, Tai SH (2010) A systematic review and metaanalysis on the therapeutic equivalence of statins. J Clin Pharm Ther 35(2): 139-151

13. Vallejo-Vaz AJ, De Marco M, Stevens CA et al (2018) Overview of the current status of familial hypercholesterolaemia care in over 60 countries-the EAS familial Hypercholesterolaemia studies collaboration (FHSC). Atherosclerosis 277:234-255

14. Reda A, Ashraf M, Soliman M, Ragy H et al (2019) The pattern of risk-factor profile in Egyptian patients with acute coronary syndrome: phase II of the Egyptian cross-sectional CardioRisk project. Cardiovasc J Africa 30(2):87-94

15. Reda A, Elbahry A, Dawood E et al (2019) Preliminary results of the Egyptian familial hypercholesterolemia research forum registry. Atherosclerosis 287:209
16. Bridgewater N and Tarrytown N (2019). Sanofi and Regeneron offer Praluent ${ }^{\oplus}$ (alirocumab) at a new reduced U.S. list price. Retrieved from: http://www.news.sanofi.us/2019-02-11-Sanofi-and-Regeneron-offer PraluentR-alirocumab-at-a-new-reduced-U-S-list-price (Accessed 15 Dec, 2019).

17. Fonarow GC, van Hout B, Villa G, Arellano J, Lindgren P (2019) Updated cost-effectiveness analysis of evolocumab in patients with very high-risk atherosclerotic cardiovascular disease. JAMA Cardiol 4(7):691-695

18. Horton JD, Cohen JC, Hobbs HH (2009) PCSK9: a convertase that coordinates LDL catabolism. J Lipid Res 50:S172-S177

19. Reiner Z (2018 Mar) PCSK9 inhibitors in clinical practice: expectations and reality. Atherosclerosis. 270:187-188

20. Wasserman SM, Sabatine MS, Koren MJ et al (2018) Comparison of LDL-C reduction using different evolocumab doses and intervals: biological insights and treatment implications. J Cardiovasc Pharmacol Ther 23(5):423432

21. Koren MJ, Lundqvist P, MENDEL-2 Investigators (2014) Anti-PCSK9 monotherapy for hypercholesterolemia: the MENDEL-2 randomized, controlled phase III clinical trial of evolocumab. J Am Coll Cardiol 63(23): 2531-2540

22. Robinson JG, Nedergaard BS, Rogers WJ et al (2014) LAPLACE-2 investigators: effect of evolocumab or ezetimibe added to moderate-or high-intensity statin therapy on LDL-C lowering in patients with hypercholesterolemia: the LAPLACE-2 randomized clinical trial. JAMA 311(18):1870-1882

23. Stroes E, Colquhoun D, Sullivan D et al (2014) Anti-PCSK9 antibody effectively lowers cholesterol in patients with statin intolerance: the GAUSS2 randomized, placebo-controlled phase 3 clinical trial of evolocumab. J Am Coll Cardiol 63(23):2541-2548

24. Nissen SE, Stroes E, Dent-Acosta RE et al (2016) Efficacy and tolerability of evolocumab vs ezetimibe in patients with muscle-related statin intolerance: the GAUSS-3 randomized clinical trial. Jama 315(15):1580-1590

25. Raal FJ, Stein EA, Dufour R et al (2015) PCSK9 inhibition with evolocumab (AMG 145) in heterozygous familial hypercholesterolaemia (RUTHERFORD-2): a randomised, double-blind, placebo-controlled trial. Lancet 385(9965):331340

26. Desai NR, Giugliano RP, Wasserman SM et al (2017) Association between circulating baseline proprotein convertase subtilisin kexin type 9 levels and efficacy of evolocumab. JAMA Cardiol 2(5):556-560

27. Roth EM, Taskinen MR, Ginsberg HN et al (2014) Monotherapy with the PCSK9 inhibitor alirocumab versus ezetimibe in patients with hypercholesterolemia: results of a 24 week, double-blind, randomized phase 3 trial. Int J Cardiol 176(1):55-61

28. Kereiakes DJ, Robinson JG, Cannon CP et al (2015) Efficacy and safety of the proprotein convertase subtilisin/kexin type 9 inhibitor alirocumab among high cardiovascular risk patients on maximally tolerated statin therapy: the ODYSSEY COMBO I study. Am Heart J 169(6):906-915

29. Cannon CP, Cariou B, Blom D et al (2015) Efficacy and safety of alirocumab in high cardiovascular risk patients with inadequately controlled hypercholesterolaemia on maximally tolerated doses of statins: the ODYSSEY COMBO I| randomized controlled trial. Eur Heart J 36(19):1186-1194

30. Moriarty PM, Thompson PD, Cannon CP et al (2015) Efficacy and safety of alirocumab vs ezetimibe in statin-intolerant patients, with a statin rechallenge arm: the ODYSSEY ALTERNATIVE randomized trial. J Clin Lipidol 9(6):758-769

31. Robinson JG, Farnier M, Krempf $\mathrm{M}$ et al (2015) Efficacy and safety of alirocumab in reducing lipids and cardiovascular events. N Engl J Med 372(16):1489-1499

32. Kastelein JJ, Ginsberg HN, Langslet G et al (2015) ODYSSEY FH I and FH II: 78 week results with alirocumab treatment in 735 patients with heterozygous familial hypercholesterolaemia. Eur Heart J 36(43):2996-3003

33. Ginsberg HN, Rader DJ, Raal FJ et al (2016) Efficacy and safety of alirocumab in patients with heterozygous familial hypercholesterolemia and LDL-C of $160 \mathrm{mg} / \mathrm{dl}$ or higher. Cardiovasc Drugs Ther 30(5):473-483

34. Hartgers ML, Defesche JC, Langslet $\mathrm{G}$ et al (2018) Alirocumab efficacy in patients with double heterozygous, compound heterozygous, or homozygous familial hypercholesterolemia. J Clin Lipidol 12(2):390-396

35. Sabatine MS, Giugliano RP, Keech A et al (2016) Rationale and design of the further cardiovascular OUtcomes research with PCSK9 inhibition in subjects with elevated risk trial. Am Heart J 173:94-101

36. Collins R, Reith C, Emberson J et al (2016) Interpretation of the evidence for the efficacy and safety of statin therapy. Lancet 388(10059):2532-2561 
37. Ridker PM, Pradhan A, MacFadyen JG, Libby P, Glynn RJ (2012) Cardiovascular benefits and diabetes risks of statin therapy in primary prevention: an analysis from the JUPITER trial. Lancet 380(9841):565-571

38. Sabatine MS, Leiter LA, Wiviott SD et al (2017) Cardiovascular safety and efficacy of the PCSK9 inhibitor evolocumab in patients with and without diabetes and the effect of evolocumab on glycaemia and risk of new-onset diabetes: a prespecified analysis of the FOURIER randomised controlled trial. Lancet Diab Endocrinol 5(12):941-950

39. Giugliano RP, Mach F, Zavitz K et al (2017) Design and rationale of the EBBINGHAUS trial: a phase 3, double-blind, placebo-controlled, multicenter study to assess the effect of evolocumab on cognitive function in patients with clinically evident cardiovascular disease and receiving statin background lipid-lowering therapy - a cognitive study of patients enrolled in the FOURIER trial. Clin Cardiol 40(2):59-65

40. Giugliano, R. P., Mach, F., Zavitz, et al (2017). Cognitive function in a randomized trial of evolocumab. N Engl J Med, 377(7): 633-643.

\section{Publisher's Note}

Springer Nature remains neutral with regard to jurisdictional claims in published maps and institutional affiliations.

\section{Submit your manuscript to a SpringerOpen ${ }^{\circ}$ journal and benefit from:}

- Convenient online submission

- Rigorous peer review

- Open access: articles freely available online

High visibility within the field

- Retaining the copyright to your article 